\title{
-5iencia Nicolaita
}

\section{Medición de temperatura en distintas partes en un motor de combustión interna a velocidades 800 y 1000 rpm}

Juan José Piña Castillo, Renato González Bernal

Para citar este artículo: Piña Castillo J.J., González Bernal R. 2022. Medición de temperatura en distintas partes en un motor de combust ión interna a velocidades 800 y 1000 rpm. Ciencia Nicolaita, número 83, 267-XX. DOI: https://doi.org/10.35830/cn.vi83.566

Ver material suplementario

Ver material suplementario

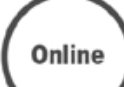

Publicado en línea, enero 2022

Envíe su artículo a esta revista 


\title{
Medición de temperatura en distintas partes en un motor de combustión interna a velocidades 800 y $1000 \mathrm{rpm}$
}

\author{
Juan José Piña Castillo*, Renato González Bernal \\ Universidad Michoacana de San Nicolás de Hidalgo, Facultad de Ingeniería Mecánica
}

\section{Historial DEL ARTÍCULO}

Recibido: 9 de septiembre de 2021 Aceptado: 8 de noviembre 2021

\section{RESUMEN}

En el presente estudio se realiza el análisis del desempeño de un motor de combustión interna de seis cilindros en posición "V", utilizando como combustible gasolina magna.Se montó un banco de pruebas para monitorear la temperatura en tres puntos: la admisión, el radiador y el escape. El objetivo del trabajo es obtener un banco de datos al monitorear la temperatura a bajos índices de 800 y 1000 revoluciones por minuto. Las pruebas se realizaron utilizando un motor de combustión interna de 4.1 litros y cuatro tiempos. En una primera etapa se llevaron a cabo pruebas utilizando gasolina magna de 87 octanos. Para el registro de las temperaturas, las pruebas se realizaron utilizando el adquisidor de datos marca Fluke de 20 canales con termopares tipo k. El análisis de los resultados muestra el desempeño del motor a las condiciones descritas. Finalmente se presenta un estudio sobre las gráficas obtenidas que servirán para la asociar el incremento de las temperaturas del motor cuando se cambie el tipo de combustible con el funcionamiento del motor. Palabras clave: Motor de combustión interna, banco de pruebas, índices de revolución.

\section{Abstract}

This study analyzes the performance of a six-cylinder internal combustion engine in "V" position, using gasoline as fuel. A test bench was set up to monitor the temperature at three points: intake, radiator and exhaust. The objective of the work is to obtain a data bank by monitoring the temperature at low rates of 800 and 1000 revolutions per minute. The tests were carried out using a 4.1-liter, four-stroke internal combustion engine. In a first stage, tests were carried out using 87-octane magna gasoline. For temperature recording, the tests were performed using a Fluke 20-channel data acquirer with k-type thermocouples. The analysis of the results shows the performance of the engine under the described conditions. Finally, a study of the graphs obtained is presented, which will be used to associate the increase in engine temperatures when the type of fuel is changed with engine operation.

KeYWORDs: Internal combustion engine, test bench, revolution indexes. 


\section{Introducción}

Desde su aparición en siglo XIX, los motores de combustión interna forman parte fundamental en la generación de potencia mecánica con aplicaciones en diversas áreas en la industria hasta nuestros días. Durante su desarrollo y modificación del mismo se ha alcanzado una la eficiencia termodinámica límite, se ha reducido considerablemente las emisiones contaminantes que forman parte de los gases de efecto invernadero, así como la especialización de las tecnologías en función de las necesidades, los entornos socioeconómicos y las condiciones ambientales donde se han de utilizar.

El exitoso empleo de los motores de combustión interna, el diseño estructural de motores estructurales y la elevación de sus índices de potencia y rendimiento fueron posibles en gran medida gracias a las investigaciones y a la elaboración de la teoría sobre el proceso de funcionamiento de los motores (González et al., 2015). Los sistemas de enfriamiento en los vehículos actuales con radiadores, son diseñados para que puedan operar en toda clase de tiempo y altitud sin tener que preocuparse del sobrecalentamiento (PF006., 2000).

Datos de la Organización Mundial de la Salud cita entre las principales fuentes de contaminación del aire provocada por partículas el transporte. Se estima que aproximadamente el $90 \%$ de las personas del mundo respiran aire contaminado y que en general los niveles más bajos de contaminación se registran en países de medidas de ingresos altos,aunque se han adoptado medidas a nivel mundial para bajar los índices de contaminación, no debemos olvidar que estas fuentes de contaminación dependen de la demanda de productos, energía y servicios que utiliza el conjunto de la sociedad (OMS.,2018). La Secretaría de Energía del gobierno mexicano muestra un aumento del $48 \%$ en la demanda de petrolíferos en los últimos 20 años, impulsando el sector del transporte e industrial, los cuales han mostrado un incremento del $75 \%$ y $36 \%$, respectivamente.

México ocupa el cuarto mayor mercado de gasolina a nivel mundial y la demanda está ligada a diferentes factores económicos, tales como: el crecimiento poblacional, el incremento, composición y eficiencia del parque vehicular, la estacionalidad y el nivel de precio prevalecientes (SENER., 2018). Las emisiones contaminantes que producen los motores de combustión interna son una preocupación a nivel mundial y en la actualidad existen regulaciones respecto a las emisiones de gases y obligan a los fabricantes a diseñar motores que cumplan con la normativa (Rafael M., 2014).

En México existe un gran potencial de recursos biomásicos para producir biocombustibles líquidos (bioetanol y biodiesel), sólidos y biogás. El potencial energético en el país según su tipo y origen son: madera de bosques nativos, residuos industriales, agrícolas de cosechas, cultivos alimenticios caña de azúcar, sorgo, aceite de palma, residuos de ganado. Existe una planta de etanol de maíz (Destilmex), que no opera porque no hay excedentes nacionales de maíz y porque el precio fijado por Petróleos Mexicanos (PEMEX) no compensa el costo de producción (Red Mexicana de Bioenergía, 2011). 


\section{Apartado experimental}

En la presente sección se describe el apartado experimental realizado para la medición de las temperaturas en los diferentes sistemas del motor de combustión interna. Se ha registrado la temperatura en el sistema de admisión, en el sistema de escape y en el radiadomnr. Estas temperaturas son de importancia ya que representan los parámetros de comparación cuando se cambie el tipo de combustible y se recomienda analizar el aumento de temperatura sobre los elementos y su tribología en otrotraba trabajo futuro. En la figura 1 se muestra el arreglo experimental para la obtención de las temperaturas a 800 y $1000 \mathrm{rpm}$, que es objetivo del presente estudio (Ver tabla 1). En las figuras 2 y 3 se muestra el motor que utilizaremos para la obtención de las temperaturas y análisis de esta investigación. En la figura 4, se muestra la posición del termopar que registró la temperatura el aire de admisión después del filtro del aire de entrada. Esta temperatura registrada nos indica las condiciones con las que entra el aire al motor y tiene relación con la densidad de masa en el interior del cilindro del motor. El termopar empleado para registrar dicha temperatura es de tipo $K$.

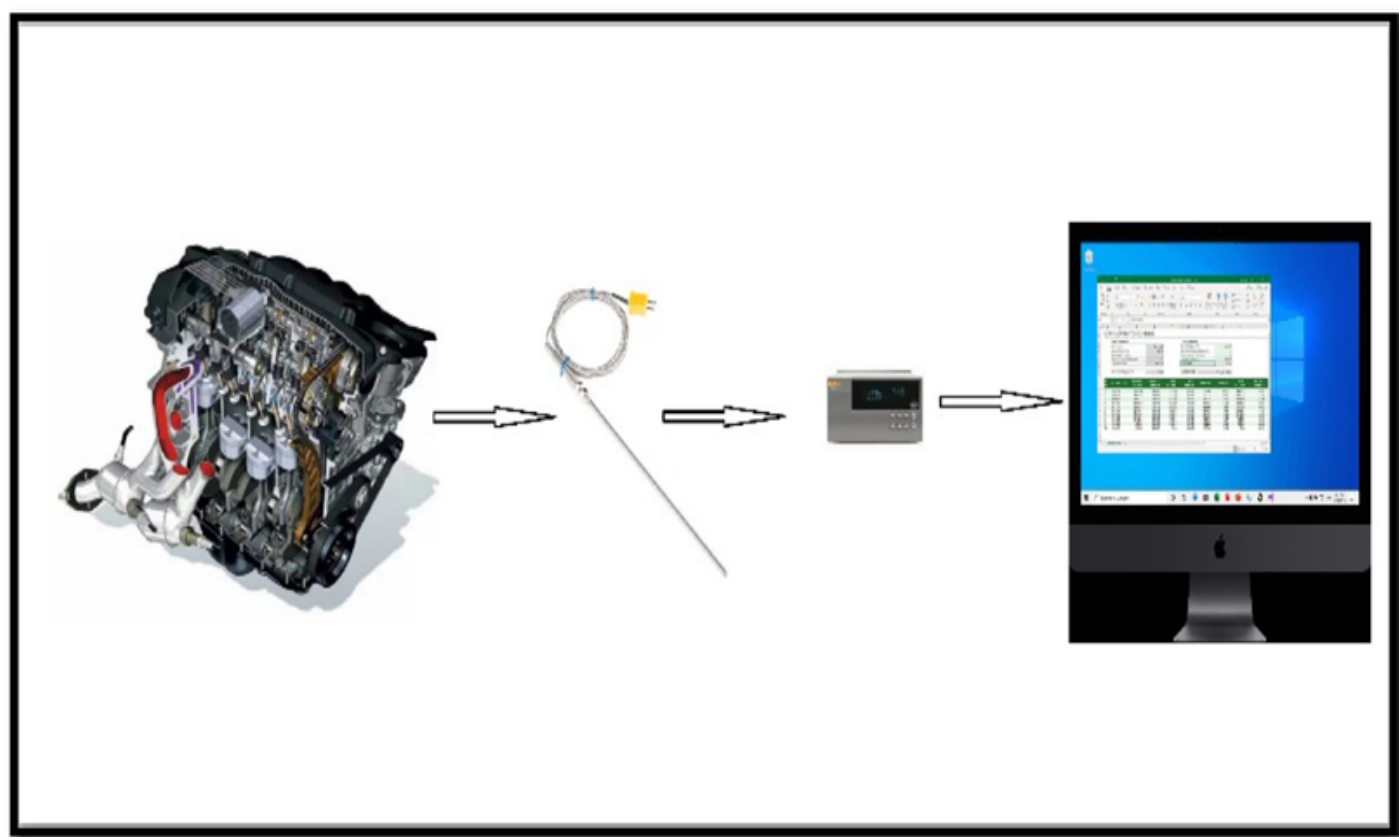

Figura 1. Arreglo experimental. 


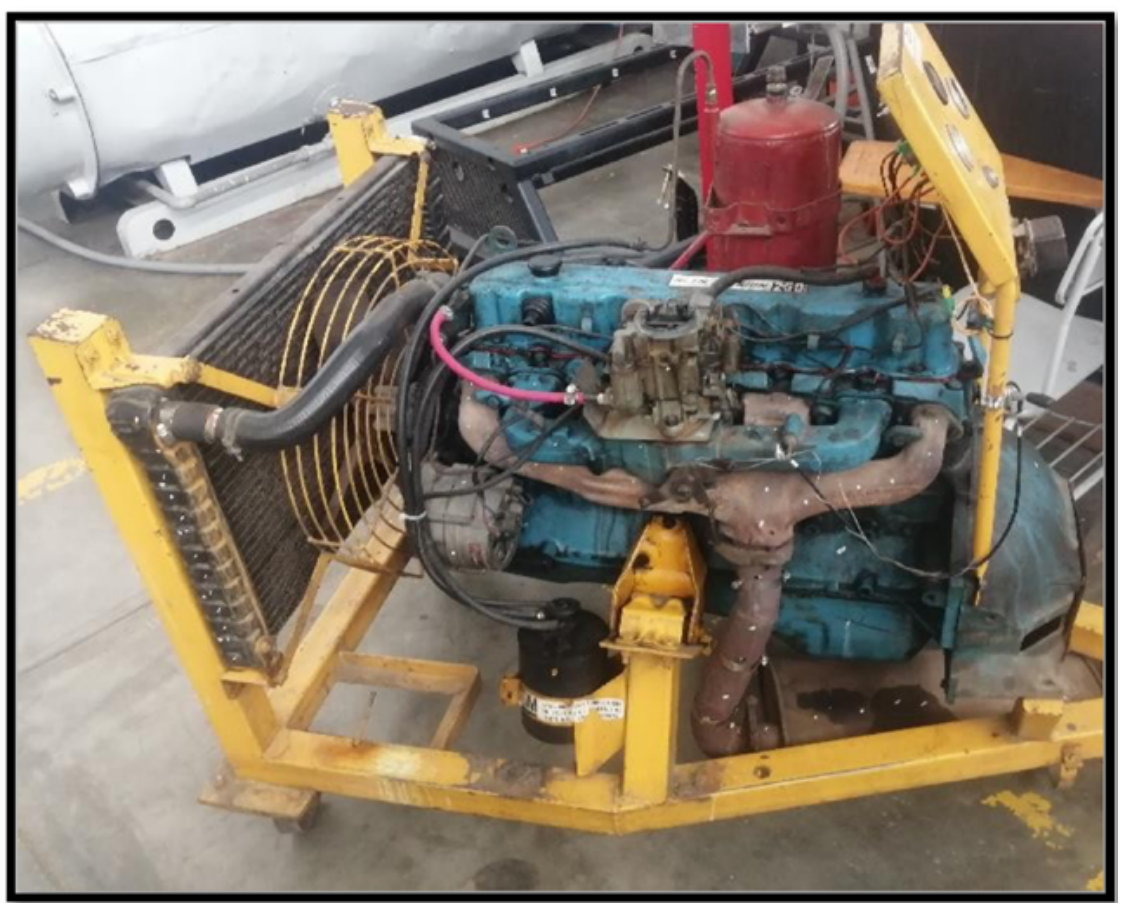

Figura 2. Vista superior del motor de $4.1 \mathrm{~L}$.

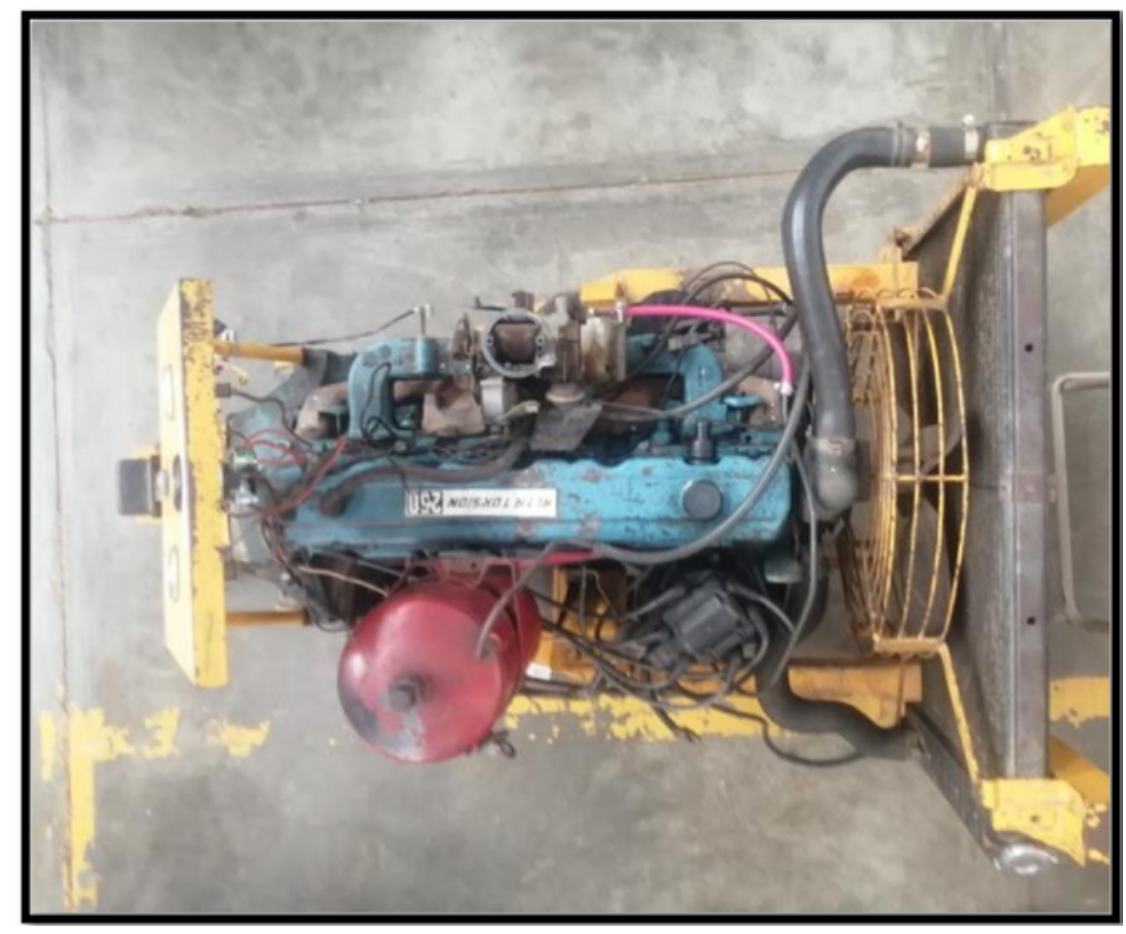

Figura 3. Vista lateral del motor de $4.1 \mathrm{~L}$. 
TABLA 1, se muestran las características del motor de combustión.

\begin{tabular}{|l|l|}
\hline Cilindro & $4097 \mathrm{cc}$ \\
\hline Número de cilindros & 6 en línea \\
\hline Diámetro & $98.43 \mathrm{~mm}$ \\
\hline Carrera & $98.66 \mathrm{~mm}$ \\
\hline Válvulas de escape & 1 por cilindro \\
\hline Válvulas de admisión & 1 por cilindro \\
\hline Suministro de combustible & Carburador \\
\hline
\end{tabular}

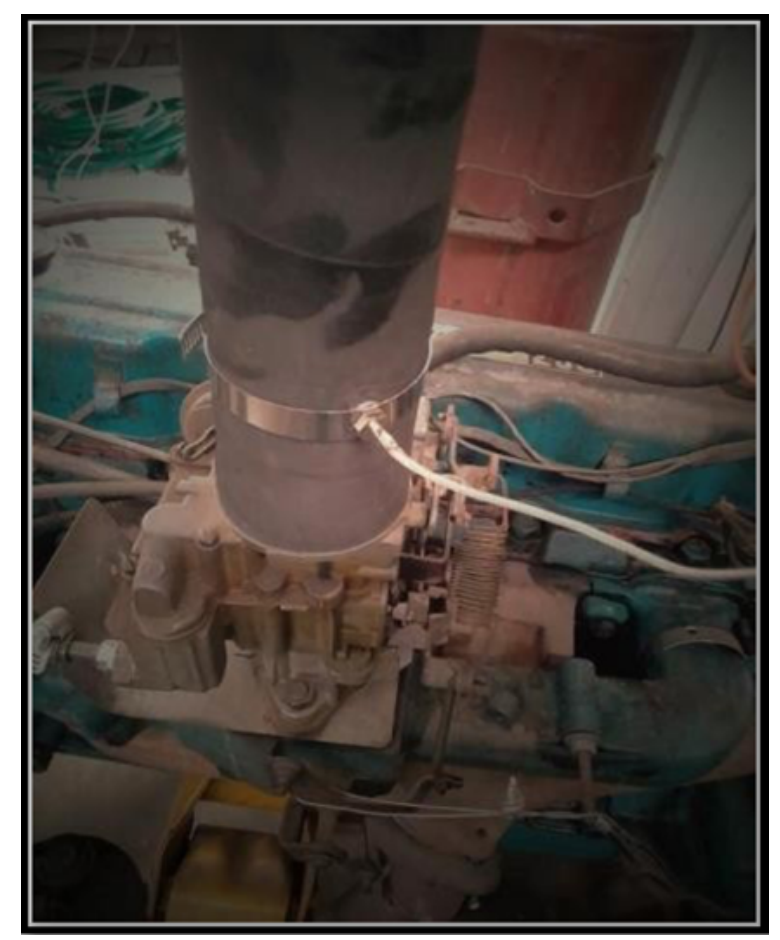

Figura 4. Termopar en la admisión.

Por otra parte, el sistema de adquisición de datos marca Fluke de 20 canales, nos hace un registro de temperatura cada segundo y lo pre senta en una tabla generada en el programa Excel.

En la figura 5 se muestra que la temperatura que se localiza a la entrada del radiador, es decir, es a la temperatura que sale del motor y que después se enfriará a su paso por el radiador. El objetivo de este punto de medir la temperatura en el interior de este, además, se muestra el punto de registro del termopar del radiador.
En la figura 6, se muestra el punto de registro de la temperatura a la salida de los gases en el escape.

En la carrera de escape, la salida del gas se da de forma espontánea y dependiendo del grado de carga que se tenga en el motor, se libera una cantidad calor, que es la que motiva que se genere por una parte en trabajo que realiza el pistón, por otra parte, una vez finalizada la carrera de trabajo, se evacuan los gases a la apertura de la válvula de escape. 


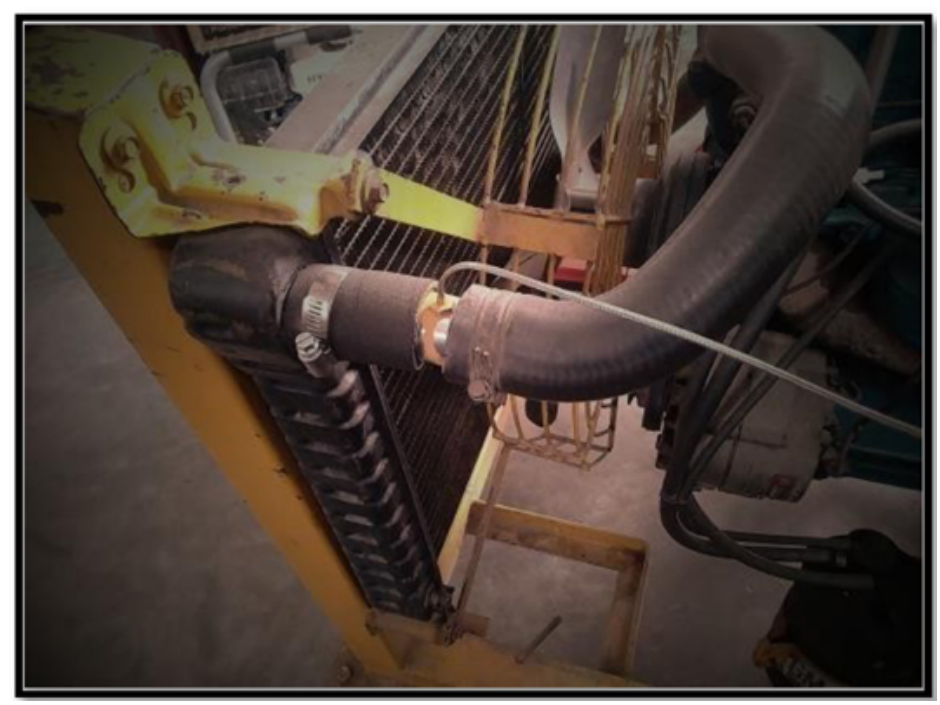

Figura 5. Termopar en la radiador.

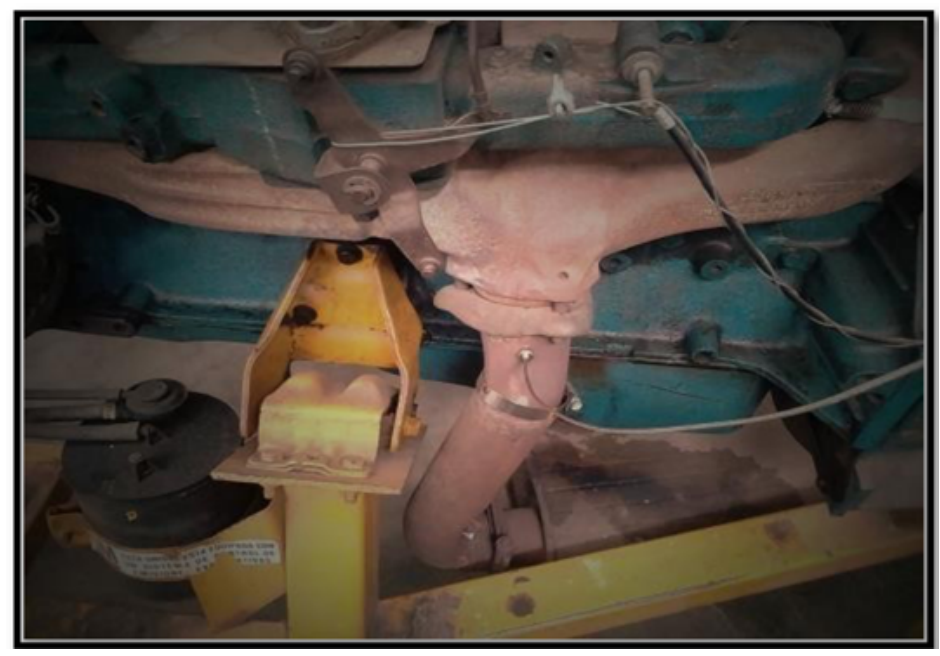

Figura 6. Termopar en el escape.

\section{Análisis de resultados}

En la presente sección se muestran los resultados obtenidos del presente trabajo de investigación. Una vez instrumentado el banco de pruebas, se obtuvieron las tablas de datos en un archivo de Excel y a partir de estos se generaron las siguientes gráficas para su análisis.

En la figura 7, se muestran las medidas de temperatura en la admisión registradas en el banco de pruebas de motor a 800 revoluciones por minuto.
En la figura 8, se muestran las medidas de temperatura en el radiador registradas en banco de pruebas del motor a 800 revoluciones por minuto.

En la figura 9 se muestran las medidas de temperatura en el escape registradas en banco de pruebas del motor a 800 revoluciones por minuto.

En la figura 10, se muestra las medidas de temperatura en la admisión registradas en banco de pruebas del motor a 1000 revoluciones por minuto. 


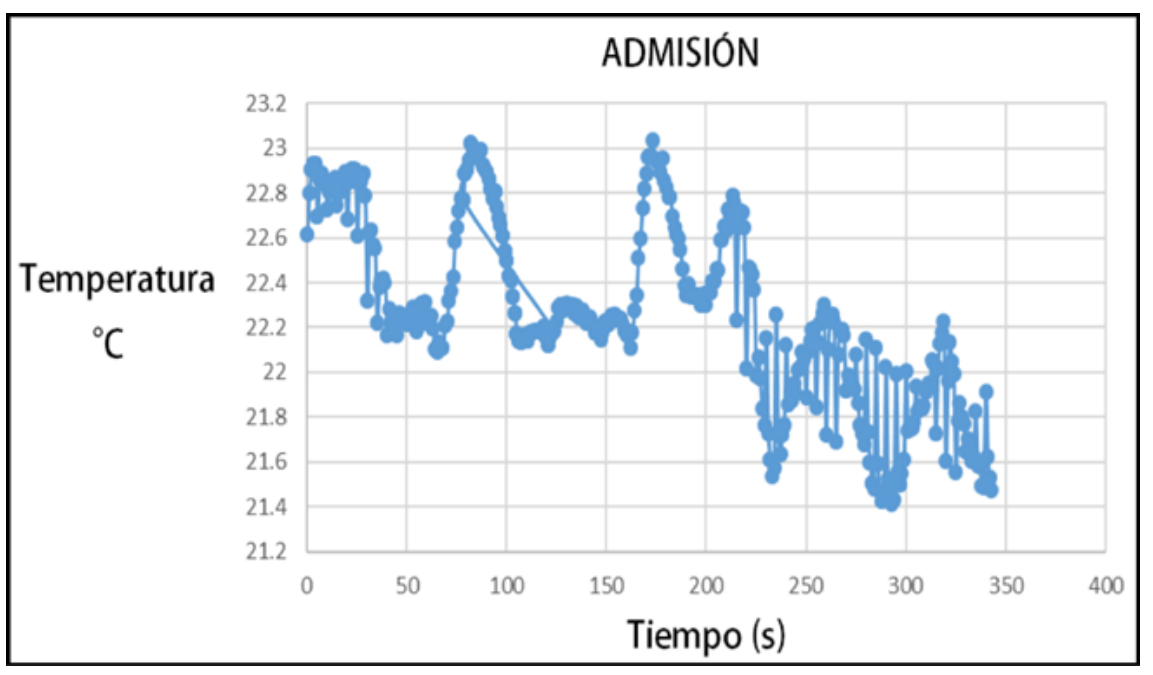

FiguRA 7. Registro de las temperaturas en la admisión registradas a $800 \mathrm{rpm}$.

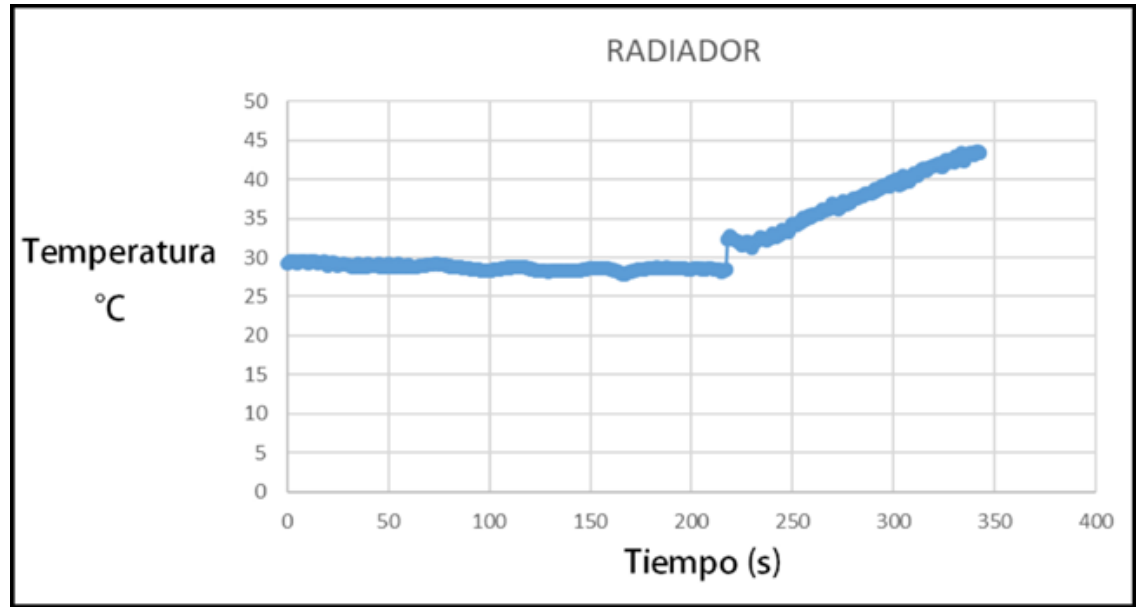

FIgURA 8. Registro de las temperaturas en el radiador registradas a $800 \mathrm{rpm}$.

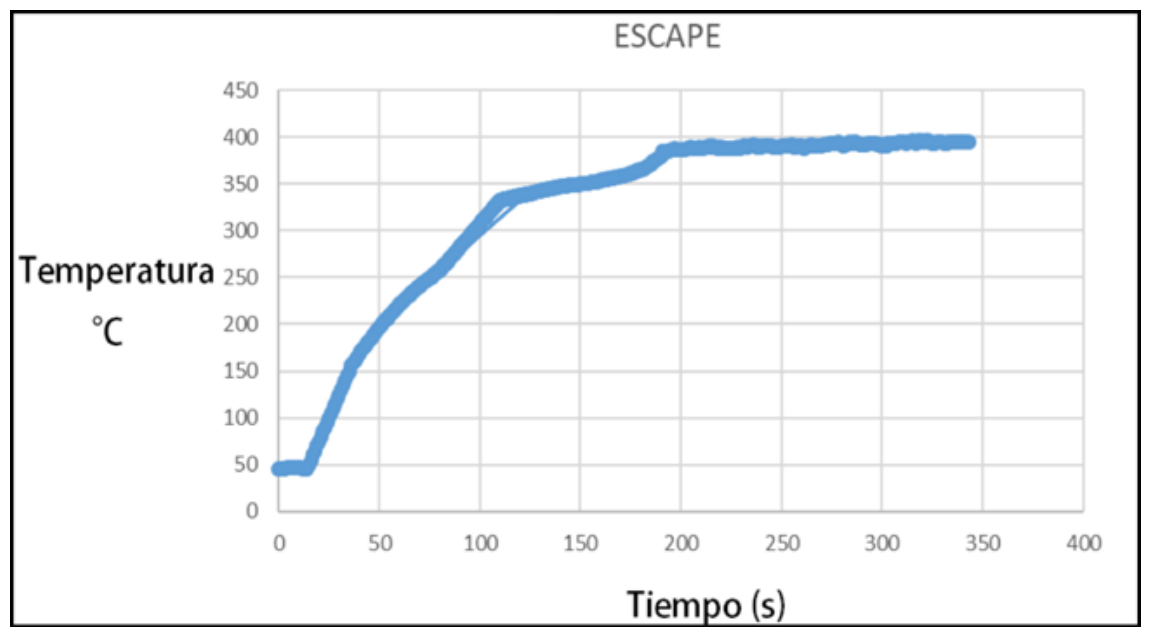

FIgURA 9. Registro de las temperaturas en el escape registradas a $800 \mathrm{rpm}$. 


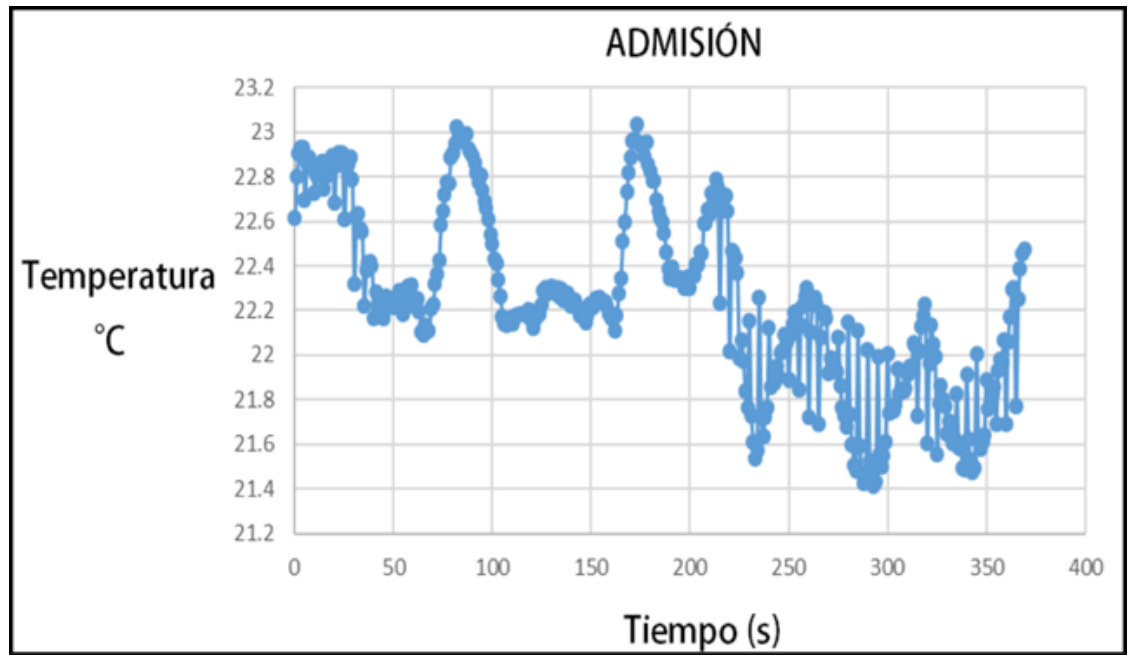

FIGURA 10. Registro de las temperaturas en la admisión registradas a 1000 rpm.

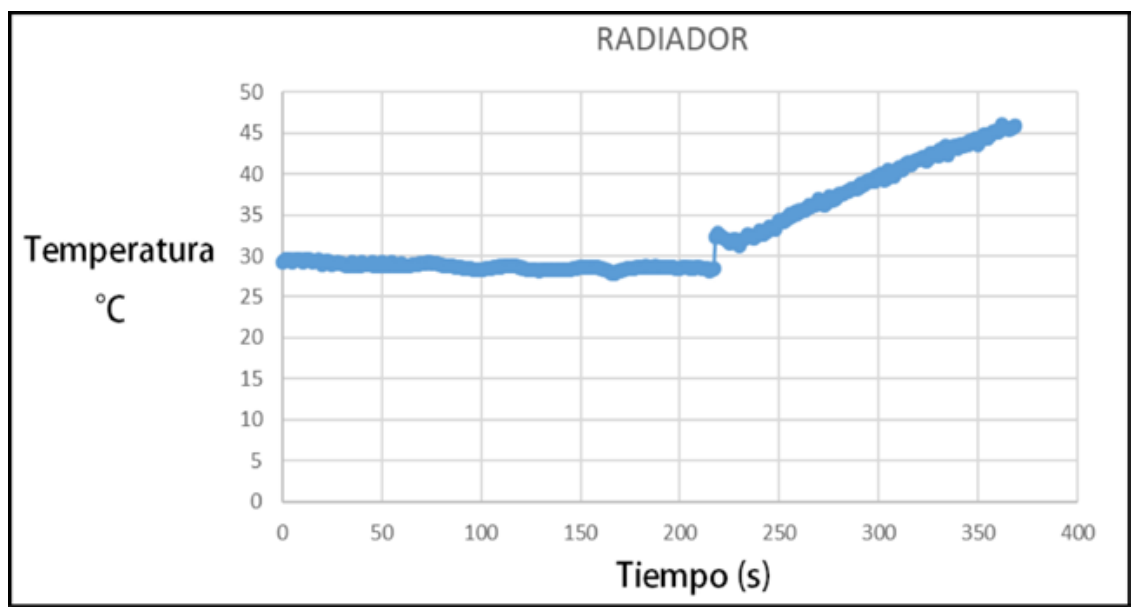

FIGURA 11. Registro de las temperaturas en el radiador registradas a $1000 \mathrm{rpm}$.

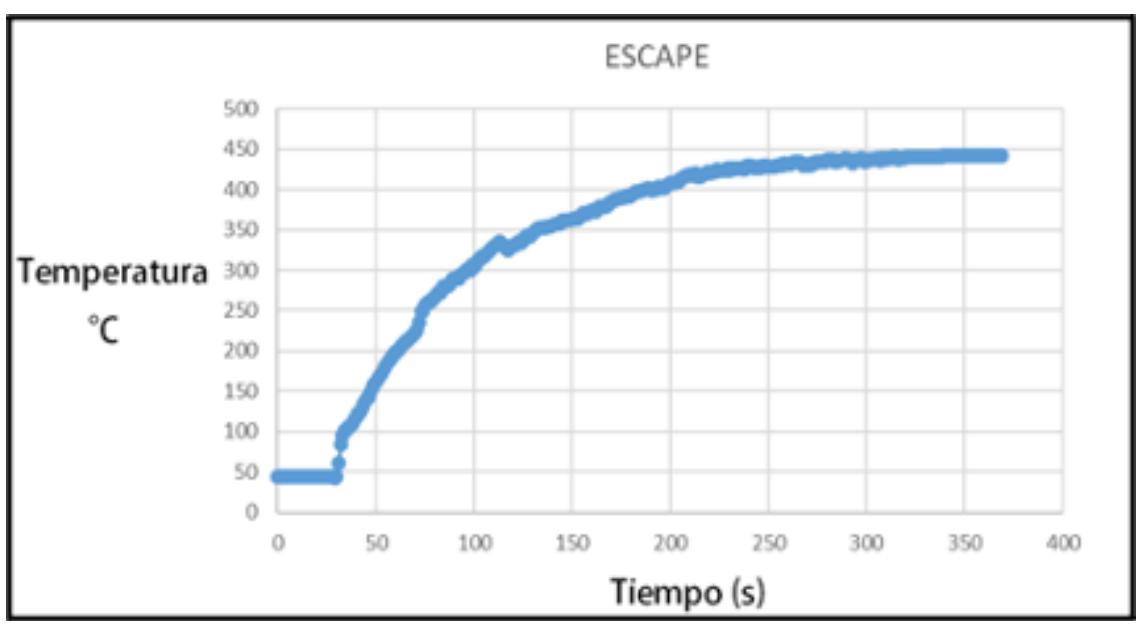

FIGURA 12. Comparación de las temperaturas en el escape registradas a 800 y 1000 rpm. 


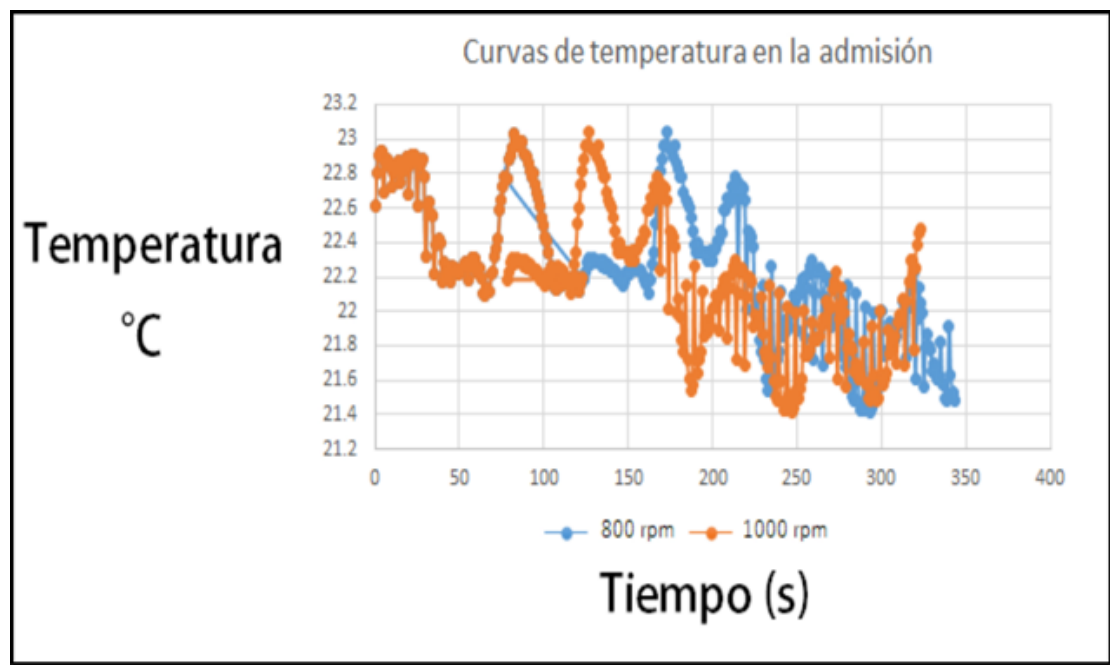

FIgURA 13. Comparación de las temperaturas a 800 y 1000 rpm

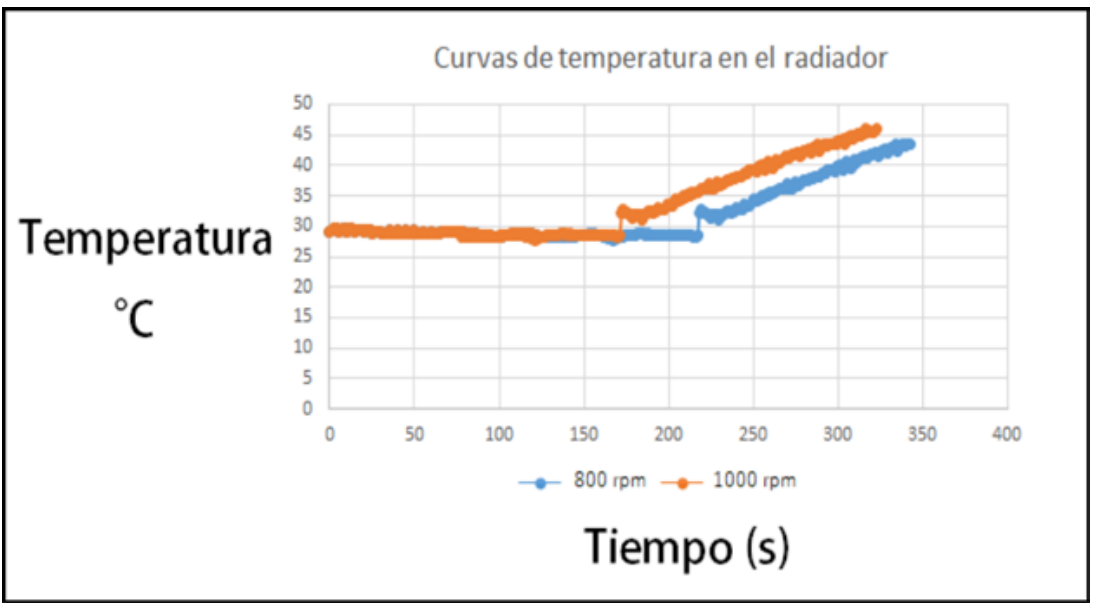

FIgURA 14. Comparación de las temperaturas a 800 y 1000 rpm

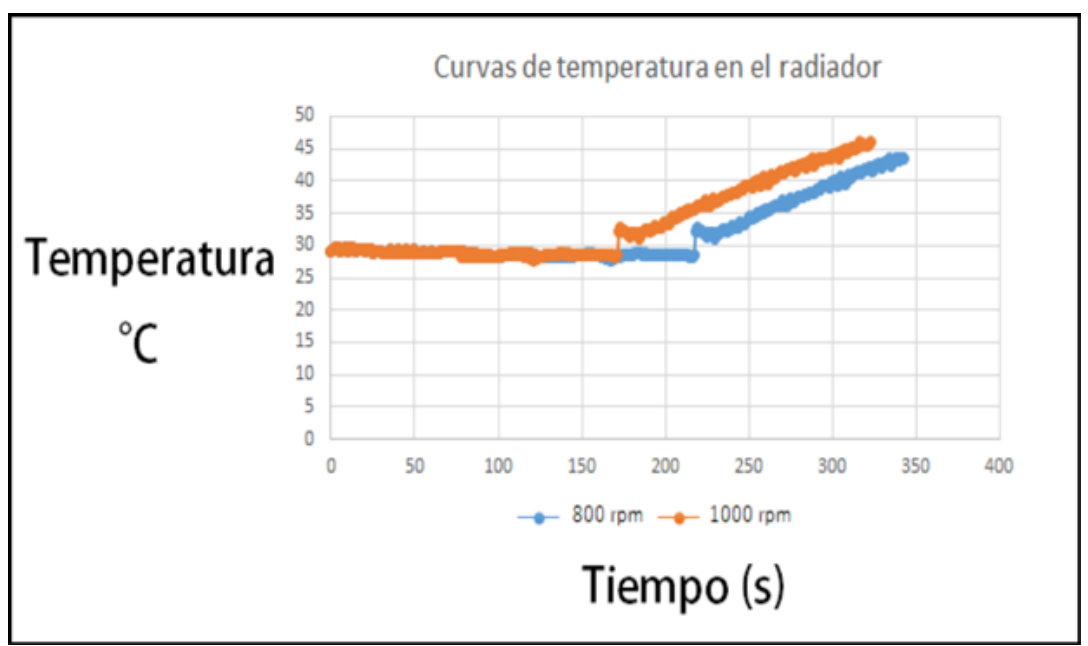

FIgURA 15. Registro de las temperaturas a 800rpm 
En la figura 11, se muestran las medidas de temperatura en el radiador registradas en banco de pruebas del motor a 1000 revoluciones por minuto.

En la figura 12 se muestran las medidas de temperatura en el escape registradas en banco de pruebas del motor a 1000 revoluciones por minuto.

En la figura 13, se observa que en la admisión al aumentar el número de revoluciones se puede observar en la gráfica una disminución de temperatura a medida que aumenta el flujo de aire debido a la compresibilidad de la mezcla aire combustible. En los casos a 800 rpm y 1000 rpm se observa un registro de temperatura alrededor de los $21.5^{\circ} \mathrm{C}$, que se puede considerar de operación para mantener la relación aire/ combustible a dichas revoluciones.

En la figura 14 las revoluciones de operación de 800 rpm y 1000 rpm son muy similares y tienen una temperatura inicial de $28.64^{\circ} \mathrm{C}$, tarda un tiempo para la estabilización aproximadamente de 3 minutos hasta la temperatura de $43.38^{\circ} \mathrm{C}$. Haciendo un análisis de las gráficas entre las revoluciones del radiador, se obtuvo una variación alrededor de $13.27^{\circ} \mathrm{C}$.

En la figura 15 para el escape se observa que para los índices de revolución de 800 y 1000 rpm inician con un registro de temperatura desde los $42^{\circ} \mathrm{C}$ y que en el menor índice de revolución de operación el motor se estabiliza aproximadamente en 3 minutos, mientras que al aumentar el índice de revolución se incrementa a 4.2 minutos.

\section{Conclusiones}

En el desarrollo del presente trabajo se ha desarrollado una metodología para la medición de temperatura en la admisión, radiador y escape en un motor de combustión interna a velocidades 800 y $1000 \mathrm{rpm}$. El interés de este procedimiento radica en generar un banco de datos que servirá para compararlos cuando el motor trabaje con otros tipos de combustible, además, de proponer una modificación de los materiales en algunos componentes del motor con la finalidad de hacerlo más eficiente y que permita cumplir con las normas de emisión de gases tipo invernadero. Para la admisión se observó que a medida que se aumentó el número de rpm hubo una disminución de temperatura. En cuanto al radiador, dentro del 800 y 1000 rpm, el incremento de temperatura fue de $13.27^{\circ} \mathrm{C}$. Finalmente, en el escape se observó que a medida que se incrementa el número de revoluciones por minuto, se incrementó la temperatura.

Con este estudio se demuestra que es posible obtener las mediciones de temperatura del motor de combustión interna con las condiciones de combustible utilizadas.

\section{Agradecimientos}

Se agradece al Programa Estímulos al Desempeño del Personal Docente, por el apoyo recibido de la Coordinación de la Investigación Científica y a la Revista Ciencia Nicolita de la Universidad Michoacana de San Nicolás de Hidalgo.

\section{Blibliografía}

Carreras, Ramón, Antonio, and Comas, Ángel Carreras. 1994. Motores de COMbUStión INTERnA: Fundamentos. EDITORIAL UPC.

EdWARd F. Obert. 1999. MOtORes DE COMBUSTIÓN INTERNA ANÁlISIS Y APLICACIONES. 2DA Edición, Editorial CECSA. Francis J. A. 2003. Desarrollo y validación de UNA METODOLOGÍA PARA CARACTERIZAR TÉRMICAMENTE RADIADORES AUTOMOTRICES. HTTPS:// TESIS.IPN.MX/BITSTREAM/HANDLE/123456789/1642/ ABUGABER.PDF?SEQUENCE $=1 \&$ ISALLOW ED $=Y$

GonzÁlez Giraldo O. E. 2015. Modelado termoDINÁMICO Y ESTRUCTURAL DE UN MOTOR DE COMBUSTIÓN INTERNA ALTERNATIVO. HTTPS://CORE. AC.UK/DOWNLOAD/PDF/71398518.PDF

Kiat Ng, H. K. 2013. Advances in Internal Combustion Engines and Fuel Technologies. INTECHOpEN. 
Organización Mundial de la Salud. https:// WWW.WHO.INT/ES/NEWS/ITEM/O2-05-2018-9-OUTOF-10-PEOPLE-WORLDWIDE-BREATHE-POLLUTEDAIR-BUT-MORE-COUNTRIES-ARE-TAKING-ACTION

OsSerain N. 2018. NuEVE DE CADA DIEZ PERSONAS EN EL MUNDO RESPIRAN AIRE CONTAMINADO. (6) Organización Mundial de la SAlud. htTps:// WWW.WHO.INT/ES/NEWS/ITEM/O2-05-2018-9-OUTOF-10-PEOPLE-WORLDWIDE-BREATHE-POLLUTEDAIR-BUT-MORE-COUNTRIES-ARE-TAKING-ACTION

Prof. F. Payti, Prof. J. M. Desantes. 2011. Motores de Combustión Interna Alternativos. EditoRIAL UPV.

Rafael M. M. Y. 2014. Caracterización de UN MOTOR DE COMBUSTIÓN INTERNA DE CON DOS TIPOS DE COMBUSTIBLE. HTTPS://WWW.IMT.MX/ARChivos/Publicaciones/PublicacionTecnica/ PT417.PDF

Red Mexicana de Bioenergía A.C. 2011.

La Bioenergía en México, SituaCión ACtUAL Y PERSPECTIVAS. HTTPS://REMBIO.ORG.MX/WP-CONTENT/UPLOADS/2014/12/CT4.PDF

Secretaría de Energía, 2018. Diagnóstico de la INDUSTRIA DE PETROLÍfEROS EN MÉXICO. HTTPS://wWW.GOB.MX/CMs/uploads/attachment/ FILE/416899/PARTE_1_VF.PDF

Simón J. Fygueroa S, Jesús O. Araque M. 2015. El Proceso de Combustión en Motores de ComBUStión Interna. Editorial Venezolana $1^{\text {a }}$ EDICIÓN. 